\title{
Présentation \\ Les notes d'auditeurs : un nouvel objet pour la linguistique et son histoire
}

\author{
Giuseppe D’Ottavi (UMR 8132 ITEM, Paris) \\ Pierre-Yves Testenoire (Université Paris-Sorbonne/UMR 7597 HTL)
}

Ce numéro consacré aux notes prises par des linguistes aux leçons de leurs maîtres se situe à la confluence de deux perspectives de recherche actuelles en sciences du langage : d'une part, un ensemble de travaux relatifs à la description du discours scientifique; d'autre part, un courant de recherche qui s'intéresse à la dimension processuelle de l'écriture en sciences humaines et sociales.

\section{Le discours scientifique}

Les recherches sur le discours scientifique, entendu ici au sens de «discours produit dans le cadre de l'activité de recherche à des fins de construction et de diffusion du savoir » (Rinck, 2010 : 428), ont connu ces vingt dernières années un développement conséquent. Issus de questionnements didactiques et de linguistique appliquée, les interrogations se sont orientées, dans une perspective plus théorique, sur les caractéristiques linguistiques du discours scientifique. À côté d'une littérature prescriptive et à visée didactique, le discours scientifique fait l'objet d'analyses descriptives empruntant des approches variées. Ce sont aussi bien les outils de l'analyse du discours (Maingueneau \& Cossutta, 1995), de la textométrie et de la linguistique de corpus (Valette, 2006; Tutin, 2007 ; Rastier, 2011 : 161-190; Tutin \& Grossmann, 2013), ou de la perspective énonciative et rhétorique (Rabatel, 2004 ; Boch \& Rinck, 2010 ; Grossmann, 2010) qui sont mobilisés sur ce type de discours. La portée de ces travaux dépasse, en outre, le champ disciplinaire strict des sciences du langage puisqu'ils intéressent, de manière réflexive, les épistémologues et les historiens des sciences (Neveu, 2008 ; Waquet, 2010).

Ce champ de recherche, extrêmement productif, accorde aujourd'hui une importance centrale à la notion de genre. Dans le sillage des Research Genres Studies (Swales 1990 et 2004) qui s'attachent aux traits linguistiques et stylistiques des formes de production scientifique contemporaine, l'article scientifique est incontestablement le genre le plus étudié (v. Poudat, 2006 ; Rinck, 2006 et 2010 pour un état des lieux bibliographique sur le sujet), mais l'on compte aussi de nombreux travaux sur la proposition de communication (Barré de Miniac, 2004), le résumé de communication (Torni \& Trabal, 2006 ; Boch \& Pons-Desoutter, 2010), l'abstract d'article (Swales \& Van Bon, 2007 ; Suomela-Salmi, 2009), le compte rendu (Salager-Meyer, Alcaraz-Ariza \& Pabón, 2007, 2010), le rapport de soutenance de thèse (Dardy, Ducard \& Maingueneau, 2002)... Si les productions écrites sont privilégiées, certaines études portent aussi sur d'autres formes de communication du savoir, longtemps présentes dans le milieu savant (v. par exemple Mazzolini, 1993), parmi lesquelles les genres universitaires oraux, comme la conférence (Goffman, 1987) ou la communication scientifique de congrès (Rowley-Jolivet, 1997 ; Rowley-Jolivet \& Carter-Thomas, 2005).

Moins étudié que l'écrit, le pan oral du discours scientifique reste d'autant plus à explorer qu'il a fait l'objet d'une réévaluation récente de la part des historiens des sciences. Ainsi l'historienne Françoise Waquet, dans deux ouvrages consacrés l'un à la parole savante (2003), l'autre à la relation mâtre-disciple dans la civilisation occidentale de l'imprimé (2008), a démontré que du XVIe au XXe siècle les formes de l'oralité savante se sont multipliées et diversifiées. Non seulement l'imprimé n'a pas tué l'oralité et la relation interpersonnelle dans le monde savant, mais celles-ci ont été investies - et continuent de l'être 
par les chercheurs - d'une haute valeur cognitive dans la formation et la transmission des connaissances. À côté d'une «oralité informelle » faite de discussions dans les laboratoires, les couloirs d'Université, les allées des congrès ou au téléphone, Françoise Waquet distingue cinq formes historiquement attestées d'«oralité formelle». Ces sont celles (1) de l'enseignement théorique de la leçon magistrale, (2) de l'enseignement pratique du séminaire, (3) de la disputatio et de ses avatars modernes dont relèvent la leçon d'agrégation ou la soutenance de thèse, (4) de l'oralité des séances d'Académie et des sociétés savantes, centrale dans la vie intellectuelle de l'Ancien Régime, enfin, (5) celle, depuis le milieu du XIXe siècle, des congrès scientifiques, des small conférences et des sessions posters. Chacune recouvre des formes d'oralité ritualisées et codifiées qui constituent de véritables genres et que l'on peut, à ce titre, historiciser (v. par exemple l'étude de Waquet, 2010 : 197-232 sur le genre de la session poster) et décrire en synchronie.

Ce sont les formes (1) et (2) de cette oralité savante qui nous intéresseront ici. Le cours universitaire, tel que le modèle humboldtien va progressivement l'imposer à partir du XIXe siècle, se conçoit comme un lieu d'articulation de la recherche scientifique et de l'enseignement. En cela, le cours universitaire, qu'il soit pratique ou théorique, relève pleinement du discours scientifique ; il dépasse le cadre strict des recherches sur l'éducation car il est un lieu, non seulement de transmission, mais aussi, via le processus de didactisation qui lui est constitutif, un lieu de formation du savoir scientifique. À ce titre, le cours universitaire a bénéficié de l'intérêt récent d'historiens (Bruter, 2008a et 2011) qui ont étudié comment les contraintes matérielles et sociales déterminent le rôle des cours dans l'économie de la production et la circulation des savoirs à une époque donnée. Objet didactique, le cours mérite aussi d'être appréhendé comme un objet historique et linguistique.

Pour l'étude de cet évènement, par nature évanescent, différents types de documents sont à disposition du chercheur. Si des documents multimédia (les diaporamas) et des enregistrements sonores ou audiovisuels sont disponibles pour la période récente (par exemple v. dernièrement Bellon, 2012 ou Derrida, 2013), les documents écrits sont les plus nombreux. Leurs nombres sont variables d'un cours à l'autre, et leurs statuts hétérogènes : documents publics (affiches, programmes, résumés de cours, sténographies de professionnels publiées), semi-publics (polycopiés et brochures distribués aux étudiants) ou privés (notes des professeurs, notes d'auditeurs). C'est par le biais de ces derniers documents, et plus précisément des notes prises par des auditeurs, que nous entendons aborder cet objet qu'est le cours.

\section{L'écriture des linguistes}

C'est Sylvain Auroux (1994) qui a posé l'écriture au fondement d'une « première révolution technologique » : la technique de l'écriture aurait conditionné l'émergence et la formation de ce qu'on appelle la «rationalité scientifique». Ses thèses prolongent, en les adaptant à l'histoire des idées linguistiques, les observations menées dans les années 1970 par les anthropologues Bruno Latour et Steve Woolgar ([1979] 1988) sur la prégnance de l'activité d'écriture dans le cadre de la recherche scientifique, ainsi que les positions de Jack Goody (1978) concernant l'influence de l'écriture, en tant que moyen de stockage et d'aliénation de l'information, sur les modes de la pensée. Si l'on admet avec ces auteurs que le développement de la science s'accompagne historiquement de l'écriture, le statut de cette dernière ne se limite pas à la formalisation des résultats, mais participe bien du processus de conceptualisation. C'est pourquoi, ces trente dernières années, historiens, philosophes et sociologues des sciences ont progressivement ajouté à l'étude des textes, observatoires de la circulation et de la validation des connaissances, la prise en compte d'écrits qui dans leur 
diversité - notes de terrain, cahiers de paillasse, annotations d'ouvrages, brouillons... témoignent de la science en train de se faire. Des linguistes (Achard, 1994 ; Mondada, 2005 ; Doquet-Lacoste, 2009; Fenoglio, 2010) ont souligné l'intérêt de «ces écrits intermédiaires » et la contribution que peut apporter l'approche énonciative à la description du processus de conceptualisation scientifique. Cette perspective bénéficie, en outre, des apports de la génétique textuelle (Grésillon, 1994 ; De Biasi, 2011) qui élargit, ces dernières années, ses investigations de son champ littéraire d'origine aux processus d'écriture des sciences dites « exactes » (Barberousse \& Pinon, 2003) et des sciences humaines (D'Iorio \& Ponton, 2003 ; Fenoglio, 2007, 2012).

Les recherches sur l'écriture scientifique dans sa dimension productive sont néanmoins contraintes par une situation archivistique différente de celle que connaissent les manuscrits littéraires. Comme le fait remarquer de Biasi (2003), les manuscrits scientifiques n'ont pas été investis de la même valeur symbolique accordée historiquement aux manuscrits littéraires. Dès lors, les politiques de patrimonialisation de ce type d'archives ont été menées à une date bien plus tardive (v. Charmasson, 2006), ce qui expliquela quantité moins importante de manuscrits de savants conservés.

Pour autant, les fonds d'archives scientifiques déjà explorés dessinent un nouveau champ d'investigation pour la génétique textuelle. Tel est le cas spécifiquement des manuscrits de linguistes. L'équipe "Génétique et théories linguistiques » de 1'Institut des Textes et Manuscrits modernes (ITEM, Paris) animée par Irène Fenoglio de 2007 à 2017 a amorcé un travail de recherche sur les spécificités de l'écriture des linguistes. Si, d'un point de vue historiographique, c'est le cas de Saussure qui a le premier attiré l'attention sur les manuscrits de linguistes (Godel, 1957), c'est avec le fonds d'archives d'Emile Benveniste qu'une campagne d'étude systématique sur les méthodes de travail du linguiste et sur les liens entre acte graphique, acte scriptural et conceptualisation a été entreprise (Fenoglio, 2009, 2011 ; Laplantine 2011; Ono, 2012). Les études saussuriennes suivent de très près, commençant à leur tour à projeter sur Saussure des perspectives et des méthodologies proprement génétiques (Testenoire, 2010, 2013 ; Sofía, 2011, 2012, 2015 ; D’Ottavi, 2012). Les travaux d'inventaire et d'exploration de nouveaux fonds d'archives qui sont actuellement en cours (Chepiga, 2012; Fenoglio \& Puech, 2012 ; Chepiga \& Sofía, 2014; Testenoire, 2015, 2016 ; Chidichimo, 2016) ouvrent dans ce domaine de nombreuses perspectives. Par la confrontation d'un nombre croissant de fonds d'archives, il s'agit à terme d'identifier des habitus de travail (Fenoglio, 2010), d'esquisser une typologie des méthodes des linguistes et de déterminer de manière sérielle les spécificités des processus d'écriture propres aux sciences du langage et à leurs activités afférentes (étude de terrain, constitution d'un dictionnaire, écriture d'une grammaire...).

Dès à présent, des récurrences se font jour entre les fonds d'archives. Ainsi, dans la plupart des fonds explorés, des notes de cours des linguistes prises aux leçons de leurs maîtres ont été conservées. Notes de Saussure aux cours d'Osthoff et de Windisch, de Bally aux cours de Saussure, de Meillet aux cours de Havet, de Benveniste aux cours de Meillet, de Redard aux cours de Benveniste... : ces documents présentent un intérêt évident pour l'histoire de la linguistique comme discipline enseignée, chacun des scripteurs devenant par la suite professeur. Ces notes dessinent aussi des chaînes de transmission de méthodes et de concepts qui donnent corps à ce que l'historiographie désigne par les catégories problématiques d' « écoles », de «courants » ou de «traditions » linguistiques. C'est ainsi que dans le cadre de l'histoire dite «externe » ou «sociale» de la linguistique (Chevalier \& Encrevé, 1984 ; Chevalier, 1992 ; Bergounioux, 1990, 2013 ; Frýba-Reber, 2013 ; Décimo, 2014), les notes prises par des linguistes majeurs aux leçons de leurs maîtres constituent un observatoire privilégié des processus de transmission des savoirs linguistiques à l'époque contemporaine. 


\section{Les notes prises aux cours de linguistique : quels observables ?}

Les notes de cours prises par les savants sont des témoins des leçons de leurs maîtres, en aucun cas leur reflet. Ce n'est donc pas la question d'une hypothétique fidélité à la parole magistrale qui guidera leurs études, mais celle des opérations scripturales qui président aux processus de formation, de transmission et d'appropriation des concepts linguistiques. Il convient, pour cela, de rendre compte de la spécificité de ces documents.

Écrits en temps contraint, en interaction avec une parole enseignante, les notes de cours sont le produit d'une activité rédactionnelle complexe : la prise de notes. Entendue comme une «activité cognitive réalisée par des noteurs pour stocker par écrit des informations lues ou entendues qu'ils jugent importantes ou pertinentes » (Piolat, 2006 : 7), la prise de notes a fait l'objet, ces dernières années, de nombreuses études de psychologues et de linguistes qui ont souligné la diversité de ses usages et de ses produits (Parpette, 2001 ; Piolat 2003, 2004, 2006 ; Branca-Rosoff, 2006...). Toutes les traces matérielles conservées sur ces documents ne peuvent néanmoins être attribuées au processus de notation effectué durant la leçon. Certaines traces écrites relèvent du temps du cours, pour reprendre la distinction opératoire proposée par Annie Bruter entre leçon et cours : «Dans la langue actuelle, cours s'entend aussi bien au sens de séquence d'enseignement, c'est-à-dire la leçon, qu'en son sens originel de parcours d'un domaine du savoir, nécessitant par conséquent une suite de leçons. Or l'observation d'une leçon n'est pas équivalente à celle d'un cours dans son ensemble » (Bruter, 2008b : 5). De fait, si l'étude du cours englobe celle de la leçon, elle ne saurait s'y borner : elle «doit nécessairement prendre en compte la série entière des leçons professées, ainsi que leur amont et leur aval»(ibid.). Si l'amont - la préparation du cours par l'enseignant - ne nous intéressera pas spécifiquement ici, l'aval, en revanche, parce qu'il met en jeu les notes prises par l'auditeur, sera l'objet de notre attention. Le travail en aval de la leçon observable dans les notes de cours obéit à une pluralité d'objectifs : travail de l'étudiant en vue d'un examen, correction à partir de notes prises par un autre auditeur, remaniement en vue d'une édition du cours... Les opérations d'écriture qui en découlent peuvent aller d'une simple formalisation logique du déjà écrit (soulignements, titrage, numérotation de séquence) à de nouvelles campagnes d'écriture d'ampleur variée: annotations complémentaires, déplacements, réaménagement voire réécriture des notes. Ces différentes configurations impliquent, outre la multiplicité des strates d'écriture, une potentielle pluralité de scripteurs. Les notes peuvent ne présenter que l'écriture d'un seul preneur de notes (v. par exemple Gambarara \& Mejía Quijano, 2005 ou Murano, 2013), ou celle-ci peut se mêler à l'écriture d'un autre auditeur ou d'un membre du public élargi du cours auquel ces notes auraient été transmises (v. la découverte de Vincent, 2013), voire à celle du professeur pour la correction d'exercices (v. D'Ottavi, 2013) ou pour la stabilisation d'un texte en vue d'une publication (v. Testenoire dans ce numéro au sujet des notes de Meillet aux cours de Havet). Les notes de cours sont donc des documents complexes, potentiellement soumis à deux temporalités - le temps contraint de la leçon, et le temps long du cours - et à plusieurs instances énonciatives.

Les objectifs de ce numéro sur les notes de cours prises par des linguistes sont les suivants :

\section{- Analyser la formation des savoirs linguistiques}

Les notes prises à ces cours présentent une grande diversité formelle. Les facteurs de cette diversité sont multiples : contingences matérielles (acoustique de la salle, conditions de la prise de notes, support de l'écrit), objectifs du scripteur, choix du mode de notation (écriture cursive, sténographie, sténotypie, schéma...). De là découlent, d'un cahier de notes à l'autre, 
une syntaxicité variable des énoncés, une spatialisation plus ou moins linéaire de l'écrit sur la page, l'usage d'inscriptions tabulaires, de diagrammes, ou encore de procédés abréviatifs (pour les problèmes sémio-linguistiques que posent ces derniers v. Andrieux-Reix, BrancaRosoff \& Puech, 2004). L'analyse des notes de cours se heurte, en partie, aux difficultés que rencontrent les linguistes travaillant sur l'oral. Si, comme l'ont analysé C. BlancheBenveniste et C. Jeanjean (1987) ou F. Gadet (1989 : 39-57), la transcription d'un discours oral implique une inévitable «reconstruction» de ce discours, comment s'opère cette reconstruction dès lors qu'il s'agit, non plus d'enregistrer des données orales, mais de sélectionner et d'assimiler des informations dans un discours sur la langue ? On s'intéressera tout particulièrement aux formulations et aux reformulations des concepts linguistiques, ainsi qu'aux phénomènes de corrections, d'annotations a posteriori, ou à la présence d'énoncés métadiscursifs sur le discours magistral.

\section{- Étudier la genèse de la publication d'un cours}

La publication d'un cours est une pratique presque aussi ancienne que l'histoire de la pensée occidentale : une partie du corpus aristotélicien, on le sait, n'est rien d'autre que les notes de cours destinées aux auditeurs du Lycée. À l'époque moderne, l'édition de cours se fonde sur quatre sources principales : notes du professeur (par exemple, les leçons de linguistique de Guillaume, 1971-2013), documents institutionnels (résumés, brochures, polycopiés : par exemple Belardi, 1959), enregistrements (comme Culioli, 1985 ou Chomsky, 1988), et notes d'auditeurs.

Les problèmes de l'édition d'un cours à partir de notes d'auditeurs - seul cas de figure qui nous retiendra ici - prend, dans le cas de la linguistique, une dimension particulière. C'est, en effet, un tel objet - le Cours de linguistique générale de Saussure [1916] (1967) - que la linguistique au XXe siècle a érigé en lieu de mémoire pour la constitution de la discipline (Chiss \& Puech, 1999). À côté de cet exemple emblématique, d'autres notes de cours prises par des linguistes ont servi à l'établissement d'un texte édité : celles de Louis Duvau pour le Cours élémentaire de métrique grecque et latine de Havet (1886), ou plus récemment, celles de Jean-Claude Coquet, de Jacqueline Authier-Revuz et de Claudine Normand pour les Dernières leçons de Benveniste (2012), par exemple.

Envisagées selon une finalité éditoriale, les notes de cours prennent un autre statut. Elles peuvent être appréhendées comme des avant-textes du cours édité et dès lors contribuer à l'analyse de la genèse de ce genre scientifique particulier qu'est le cours publié.

- Interroger les concepts d' "école » et de «tradition » linguistiques

Enfin, l'étude des notes des linguistes aux leçons de leurs maîtres se veut une contribution à l'histoire et à l'épistémologie des théories linguistiques. En observant les mécanismes à l'œuvre dans la transmission des savoirs linguistiques, nous entendons interroger les notions d'écoles ou de traditions linguistiques, catégories utiles mais problématiques pour l'historien des sciences, et spécifiquement des sciences du langage (v. Amsterdamska, 1987 ; Servos, 1993 ; Savatovsky, 1995 ; Chiss \& Puech, 1999 ; Puech, 2015). Le cours, comme espace d'élaboration et de transmission, paraît, en effet, déterminant pour la formation d'une communauté de méthode et de pensée (v. D’Ottavi, 2015). L'approche de ces questions se veut complémentaire de celle développée par l'histoire conceptuelle. Alors que cette dernière appréhende ces phénomènes par des cooccurrences de concepts, d'exemples et d'arguments repérables d'un texte à l'autre, l'étude des notes prises par des linguistes aux leçons de leurs maîtres permet de décrire en acte un mode, parmi d'autres, de transmission d'un savoir sur les langues. La transmission des idées linguistiques est alors envisagée comme l'interaction de 
pratiques discursives - une parole enseignante et les processus scripturaux d'un auditeur accessibles via les traces conservées dans les notes de ce dernier.

\section{L'apport du numéro Le cours de linguistique. Formes, genèses et interprétations de notes d'auditeurs}

Les contributions réunies dans ce volume portent sur des cours universitaires de linguistique dispensés en langue française entre la fin du XIXe siècle et aujourd'hui. Le champ et la période considérés présentent, au regard du temps long de l'histoire des idées linguistiques, une cohérence certaine. Depuis les dernières décennies du XIXe siècle, qui voient l'institutionnalisation de la linguistique dans le système universitaire français, jusqu'aux premières décennies du XXIe siècle, le «cours de linguistique » se présente comme un objet relativement homogène et stabilisé. En dépit des changements institutionnels, de l'évolution des populations professorales et estudiantines et des innovations pédagogiques et matérielles, le « cours » reste, sur cette période, soumis à l'interaction des mêmes acteurs (un professeur et des auditeurs) et à des contraintes institutionnelles et sociales similaires (examens, programmes...). Dans ce cadre, les contributions du numéro se concentrent sur l'enseignement de cinq grands linguistes - Louis Havet, Ferdinand de Saussure, Antoine Meillet, Emile Benveniste et Antoine Culioli - et sur les notes prises par leurs auditeurs. Les choix effectués ne visent évidemment aucune représentativité. Ils sont en partie contraints par les sources archivistiques disponibles et par l'état de nos connaissances pour ce champ de recherche nouveau. Cependant, ils isolent une tradition bien identifiée, celle de la linguistique historique et comparée et de la linguistique française - ce que l'historiographie appelle parfois «l'école française de linguistique » ou «l'école de Paris »- ancrée institutionnellement à l'École Pratique des Hautes Études et dans la succession de la chaire de grammaire comparée au Collège de France. Le choix de se concentrer sur cette chaîne homogène d'enseignement est en cohérence avec l'ambition générale d'interroger le concept d' «école linguistique ». Enfin, les cas abordés dans ce numéro ne sont pas réunis par hasard : tous les savants dont l'enseignement est étudié sont des figures scientifiques pour lesquelles l'oral a joué un rôle important dans la transmission et la diffusion de leurs idées linguistiques. Leurs enseignements ont en commun d'avoir donné lieu à des cours publiés, soit de leur vivant (Havet, Culioli), soit à titre posthume (Saussure, Meillet, Benveniste).

Les contributions réunies dans ce numéro se signalent également par leur cohérence méthodologique. Chacune propose une étude de cas et une contribution originale aux problématiques du «cours de linguistique » dégagées ci-dessus. La plupart des articles réunis reposent sur un travail d'archives et exploitent des documents inédits. Tous articulent une description linguistique, parfois aussi génétique, des matériaux écrits et sa mise en perspective avec les savoirs linguistiques de l'époque. Parallèlement à l'unité thématique et méthodologique, la complémentarité des points de vue met en évidence les différentes sources d'intérêt que suscite ce type de documents. Certaines contributions insistent sur le rôle historique des cours universitaires dans le processus de disciplinarisation de la linguistique (Bergounioux), d'autres sur l'importance de l'élaboration conceptuelle effectuée dans ce cadre pour la compréhension de l'ensemble de l'œuvre théorique de tel ou tel savant (Ducard). Quatre contributions abordent directement le problème de la «mise en livre » d'un enseignement oral, soit en interrogeant les gestes scripturaux et éditoriaux qu'elle implique (Testenoire, Sofia, Fenoglio), soit en examinant les spécificités linguistiques de ces notes d'auditeurs situées à l'interface entre l'oral et l'écrit (Doquet).

La présentation des articles suit un ordre chronologique. Elle vise aussi à faire se répondre les contributions qui abordent, sous des angles différents, un même enseignement. 
L'article qui ouvre le dossier est une mise au point sur l'enseignement universitaire de la linguistique en France au XIXe siècle par un spécialiste reconnu de la question (Bergounioux, 1984, 1990, 1998...). Gabriel Bergounioux propose, dans ce panorama historique, une étude comparée du destin contrasté de l'enseignement de linguistique générale donné en même temps par Saussure et par Meillet, qu'il analyse comme un effort de synthèse à l'aube du XXe siècle. Le deuxième article, de Pierre-Yves Testenoire, constitue également une introduction générale à la problématique du cours publié dont la catégorisation comme genre est interrogée. Testenoire examine ensuite un cas particulier d'écriture collaborative, entre un professeur (Louis Havet) et un auditeur (Antoine Meillet) pour la publication d'un cours. Le cas incontournable de l'enseignement de Saussure fait l'objet de l'article suivant. Estanislao Sofía étudie un document inédit de la genèse du Cours de linguistique générale : le manuscrit de la collation effectuée par Sechehaye, revu par Bally, à partir des différentes notes d'auditeurs. La spécificité de ce document, où se superposent différentes strates chronologiques d'écriture et d'auctorialité, lui fournit l'occasion de réfléchir à l'activité de la prise de notes dans la diversité de ses pratiques. Irène Fenoglio explore un autre aspect du statut philologique et épistémologique des notes d'auditeurs. Elle revient, dans une démarche réflexive, sur son expérience d'éditrice des Dernières leçons de Benveniste pour évaluer l'importance de l'«espace épistémologique » caractéristique de ce type de documents. Elle montre que les notes d'auditeurs sont à la fois un outil privilégié pour la recomposition de l'ordre d'énonciation d'un cours et un espace de genèse théorique. Les deux derniers articles portent sur la période contemporaine et joignent à l'étude des notes d'auditeurs l'exploitation d'archives sonores. Dominique Ducard réfléchit aux spécificités de l'enseignement de Culioli à partir de ses propres notes prises en tant qu'auditeur qu'il croise avec les écrits antérieurs du linguiste. Il s'intéresse en particulier aux phénomènes d'intensité dans l'énonciation envisagés à la fois comme notion théorique et comme modalité d'exposition. Le dernier article, de Claire Doquet, traite également de l'enseignement de Culioli et constitue une ouverture sur un genre voisin, également ancien, qui présente de nombreuses similitudes avec le cours : celui de l'entretien. Son étude se fonde sur le dossier de genèse complet des Variations sur la linguistique paru en 2002. À travers les différentes étapes de la constitution du livre, elle étudie deux problèmes majeurs que pose toute écriture des «paroles » d'un maître : celui de la représentation du discours autre et celui de la mise en écrit du discours oral.

Ce numéro de Langages consacré au cours de linguistique s'inscrit, en définitive, dans le mouvement de recherche actuel sur le discours scientifique et sur l'écriture en sciences humaines. Son objectif est de mettre en évidence, dans l'attention croissante portée aux manuscrits et aux écrits quotidiens des chercheurs au sein de cadres disciplinaires variés (cf. Jacob, 2011 ; Lefebvre, 2013; Bert, 2014; Waquet, 2015), l'intérêt et l'originalité de l'approche des linguistes. L'ambition de ce numéro est de penser comme objet d'étude un type de documents - les notes prises par des auditeurs - non considérés en tant que tels jusque-là. Source de première importance pour l'histoire des idées linguistiques, ces documents soulèvent également de nombreux problèmes d'ordre linguistique - des problèmes de description, d'énonciation et de conceptualisation - qui sont au cœur des contributions de ce dossier.

\section{BIBLIOGRAPHIE}

ACHARD P. (1994), «L'écriture intermédiaire dans le processus de recherche en sciences sociales », Communications 58, 149-156. 
AMSTERDAMSKa O. (1987), School of Thought. The Development of Linguistics from Bopp to Saussure, La Haye : Mouton.

AndrieuX-ReiX N., BrancA-Rosoff S. \& Puech C. (éds.) (2004), Écritures abrégées (notes, notules, messages, codes...), Paris : Orphys.

AUROUX S. (1994), La révolution technologique de la grammatisation, Liège : Mardaga.

BARberousse A. et PINON L. (éds.) (2003), Genesis 20 : L'écriture scientifique.

BARRE DE MiniaC C. (2004), «Une pratique d'écriture scientifique : la proposition de communication », Pratiques 121-122, 199-216.

BELARDI W. (1959), Elementi di fonologia generale [1952], Rome : Edizioni dell'Ateneo.

BELlon G. (2012), Une parole inquiète. Barthes et Foucault au Collège de France, Grenoble : Ellug.

Belmar A. G. \& Bertomeu SANChez J. R. (2004), «Les cahiers d'élèves : sources pour une histoire des contenus et des pratiques de l'enseignement de la chimie », in Le cours magistral : modalités et usages (XVIe-XXe siècles), Institut français de l'éducation :

[http://www.inrp.fr/she/cours magistral/expose thenard/expose thenard_complet.htm]

Benveniste E. (2012), Dernières Leçons. Collège de France (1968-1969), éd. par J.-C. Coquet et I. Fenoglio, Paris : Seuil.

Bergounioux G. (1984), «La science du langage en France de 1870 à 1885 : du marché civil au marché étatique », Langue française 63, 7-41.

BergouniouX G. (1990), «L'enseignement de la linguistique et de la philologie en France au XIXe siècle, d'après les affiches de cours des facultés des lettres (1845-1897)», Archives et Documents de la S.H.E.S.L. $2^{\mathrm{e}}$ série, 2, 1-105.

BERGOUNIOUX G. (1998), «Science et institution : la linguistique et l'Université en France (1865-1945) », Langue française 117 (1), 22-35.

BERGOUNIOUX G. (2013), «Les conditions d'une histoire sociale de la linguistique en France aux $\mathrm{XIX}^{\mathrm{e}}$ et $\mathrm{XX}^{\mathrm{e}}$ siècles », in G. KREMNITZ (éd.), Histoire sociale des langues de France, Rennes : Presses Universitaires de Rennes, 127-138.

BERT J.-F. (2014), Qu'est-ce qu'une archive de chercheur?, Marseille, Open Edition Press. [http://books.openedition.org/oep/438]

BIASI P.-M. de (2003), «Sciences : des archives à la genèse. Pour une contribution de la génétique des textes à l'histoire des sciences », in BARBEROUSSE et PINON (2003), 1952.

BIASI P.-M.de (2011), La génétique des textes [2000], Paris : CNRS Éditions.

BlanCHE-BENVENISTE C. \& JEANJEAN C. (1987), Le Français parlé. Transcription et édition, Paris : Didier-Érudition.

Boch F. \& Pons-DesoutTer M. (2010), « Le résumé de communication », in GROSSMANN (2010), 527-549.

[http://w3.u-grenoble3.fr/lidilem/labo/file/RAC_Boch_Pons_Dessouter.pdf]

Boch F. \& RincK F. (éds.) (2010), Lidil 41 : Énonciation et rhétorique dans l'écrit de recherche.

BRANCA-RosofF S. (2006), «Littératie et prise de notes. Le primat de la fonction iconique », Pratiques 131-132, 187-198.

Bruter A. (éd.) (2008a), Histoire de l'éducation 120 : Le cours magistral XVe-XXe siècles. 1. Publics et savoirs. [http://histoire-education.revues.org.gate3.inist.fr/1035]

BRUTER, A. (2008b) « Le cours magistral comme objet d'histoire », in BRUTER (2008), 5-32.

BRUTER A. (éd.) (2011), Histoire de l'éducation 130 : Le cours magistral XIXe-XXe siècles. 2. Le cadre institutionnel et matériel. [http://histoireeducation.revues.org.gate3.inist.fr/23241 
ChARMASsOn T. (2006), « Archives scientifiques ou archives des sciences: des sources pour l'histoire », La revue pour l'histoire du CNRS, 14 [en ligne].

[https://histoire-cnrs.revues.org/1790]

Chepiga V. (2012) «La préparation d'un ouvrage inachevé : "La glottologie" de Lucien Tesnière », in FENOGLIO (2012), 87-99.

Chepiga V. et Sofia E. (éds.) (2014), Archives et manuscrits de linguistes, Louvain la Neuve : Academia-Bruylant.

Chevalier J.-C. (1992), «Philologues et linguistes dans leurs institutions », Communications $54,149-159$.

CheVAlier J.-C. \& ENCREVE Pierre (éds.) (1984), Langue française 63 : Vers une histoire sociale de la linguistique.

Chidichimo A. (2016), «Le fonds Serge Karcevski à Genève », Acta structuralica 1, 27-62 : [http://doi.org/10.19079/acta.2016.1.27]

CHISs J.-L. \& PUECH C. (1999), Le langage et ses disciplines, XIXe-XXe siècles, Bruxelles : Duculot.

Chomsky N. (1988), Language and Problems of Knowledge, Cambridge, Mass./London : MIT Press.

Culioli A. (1985), Notes du séminaire de DEA. 1983-1984. Poitiers : Université de Paris 7, Département de recherches linguistique - Université de Poitiers.

DARDY C., DUCARD D., MAINGUENEAU D. (éds.) (2002), Un genre universitaire : le rapport de soutenance de thèse, Villeneuve d'Ascq : Presses Universitaires du Septentrion.

Decimo M. (2014), Sciences et pataphysique 2. Comment la linguistique vint à Paris. De Michel Bréal à Ferdinand de Saussure, Dijon : Les Presses du réel.

DERrIDA, J. (2013), A dessein, le dessin - Suivi de "Derrida à l'improviste » par Ginette Michaud, Le Havre : Franciscopolis.

D’Iorio P. \& Ponton O. (éds.) (2003), Genesis 22 : Philosophie.

D’Ottavi G. (2012), «Genèse d'un écrit saussurien : de la 'théosophie' à une approche de la subjectivité », in FENOGLIO (2012), 129-141.

D’Ottavi G. (2013), « Il caso degli altri corsi. I quaderni di sanscrito di Henri Duchosal », in D. Gambarara \& M P Marchese (éds.), Guida per un'edizione digitale dei manoscritti di Ferdinand de Saussure, Alessandria : Edizioni dell'Orso, 55-79.

D'OtTAVi G. (2015), «Aux sources d'une école : notes de maîtres et cahiers d'étudiants », Histoire, Épistémologie, Langage 37/2, 33-52.

DoqueT-LACOSTE C. (éd.) (2009), Langage et société 127 : Écritures scientifiques : carnets, notes, ébauches.

Fenoglio I. (éd.) (2007), L'écriture et le souci de la langue. Écrivains, Linguistes: témoignages et traces manuscrites, Louvain-la-Neuve : Academia-Bruylant.

FENOGLIO I. (2009), «Les notes de travail d'Emile Benveniste : où la pensée théorique naît via son énonciation », in DoQUET-LACOSTE (2009), 23-49.

FENOGLIO I. (2010), «Conceptualisation linguistique : du manuscrit au texte. Contribution à l'étude des spécificités de l'écriture scientifique », Actes du Congrès Mondial de Linguistique Française (CMLF 2010), Paris: EDP Sciences, 1123-1139. [http://dx.doi.org/10.1051/cmlf/2010110]

FENOGLIO I. (2011), « Déplier l'écriture pensante pour re-lire l'article publié. Les manuscrits de "L'Appareil formel de l'énonciation" d'Émile Benveniste » in E. Brunet \& R. Mahrer (éds.), Relire Benveniste. Réceptions actuelles des Problèmes de linguistique générale, Louvain-la-Neuve : Academia-Bruylant, 263-304.

FENOGLIO I. (éd.) (2012), Genesis 35 : Le geste linguistique.

Fenoglio I. \& Puech C. (2012), «Le fonds Antoine Meillet », in Fenoglio (2012), 191-196. 
Fløtтum K. (2007) (éd.), Language and Discipline Perspectives on academic Discourse, Cambridge : Cambridge Scholars Publishing.

FRÝBA-REBER A.-M. (2013), Philologie et linguistique romanes. Institutionnalisation des disciplines dans les universités suisses (1872-1945), préf. G. Bergounioux, Leuven : Peeters.

GADET F. (1989), Le français ordinaire, Paris : Armand Colin.

Gambarara D. \& Mejía QuiJano C. (éds.) (2005), «Emile Constantin, "Linguistique générale, Cours de M le Professeur de Saussure, 1910-1911" », Cahiers Ferdinand de Saussure 58, 83-290.

Godel R. (1957), Les sources manuscrites du Cours de linguistique générale de $F$. de Saussure, Genève : Droz.

GofFMAN E. (1987), «La conférence», in Façons de parler [1981], Paris : Éditions de Minuit, 167-204.

Goody J. (1978), La raison graphique. La domestication de la pensée sauvage [1977], Paris : Éditions de Minuit.

GRESILLON A. (1994), Éléments de critique génétique, Paris : PUF.

Grossmann F. (éd.) (2010), Revue d'anthropologie des connaissances 4/3: L'auteur scientifique. Des rhétoriques aux épistémologies.

Guillaume G. (1971-2013), Leçons de linguistique de Gustave Guillaume, 21 volumes, éd. sous la direction de R. Valin et R. Lowe, Québec et Paris : PUL et Klincksieck.

HAVET L. (1886), Cours élémentaire de métrique grecque et latine - Rédigé par Louis Duvau, Paris : Librairie Delagrave.

JасОв C. (éd.) (2011), Lieux de savoir 2. Les mains de l'intellect, Paris : Albin Michel.

LAPlantine C. (2011), Émile Benveniste, l'inconscient et le poème, Limoges : LambertLucas.

Latour B. et WoOlgar S. (1988), La vie de laboratoire. La production des faits scientifiques [1979], Paris : La Découverte.

LEFEBVRE M. (éd.) (2013), Sciences de la Société 89: L’infra-ordinaire de la recherche. Archives, mémoires et patrimoine scientifique.

MAingueneAu D. \& CossutTA F. (1995), «L'analyse des discours constituants », Langages $117,112-125$.

Mazzolini R. G. (éd.) (1993), Non-verbal Communication in Science prior to 1900, Firenze : Leo Olschki.

MONDADA L. (2005), Chercheurs en interaction : comment émergent les savoirs. Lausanne : Presses polytechniques et universitaires romandes.

MuRANO F. (2013), Il corso di "Étymologie grecque et latine" (1911-1912) di Ferdinand de Saussure negli appunti di Louis Brütsch, Alessandria : Edizioni dell'Orso.

Neveu F. (2008), "Réflexions sur la forme du discours linguistique », Actes du Congrès Mondial de Linguistique Française (CMLF 2008), Paris : EDP Sciences, 1069-1082 : [http://dx.doi.org/10.1051/cmlf08336]

ONO A. (2012), «"Le nom c'est l'être". Les notes préparatoires d'Emile Benveniste à l'article "La Blasphémie et l'euphémie" », in FenOGLIO (2012), 77-86.

PARPETTE C. (2001), « Le cours magistral, un discours oralographique : effet de la prise de notes des étudiants sur la construction du discours de l'enseignant » in R. Gauthier et A. Meggori (éds.), L'oralité dans l'écrit et réciproquement, Actes du 22e Colloque d'Albi Langages et signification (Juillet 2001), Toulouse: CALS-CPST, 261-266: [http://lesla.univ-lyon2.fr/sites/lesla/IMG/pdf/doc-189.pdf]

Piolat A. (2003) (éd.), Arob@se 7 (1-2) : La prise de notes en langue première et en langue seconde. 
Piolat A. (2004), «La prise de notes : écriture de l'urgence», in A/ Piolat (éd.) Écriture. Approches en sciences cognitives, Aix en Provence: Publication de l'Université de Provence, 205-229.

Piolat A. (2006), La prise de notes [2001], Paris : PUF.

PhILIPPE G. (éd.) (2014), Genesis 39 : Avant-dire. La genèse écrite des genres oraux.

POUDAT C. (2006), Étude contrastive de l'article scientifique de revue linguistique dans une perspective d'analyse des genres, Thèse de doctorat, sous la direction de G. Bergounioux, Université d'Orléans.

[http://www.revue-texto.net/Corpus/Publications/Poudat/Etude.html]

PuEch C. (éd.) (2015), Histoire Epistémologie Langages 37/2: "Faire école » en linguistique au XXe siècle : l'école de Genève.

RABATEL A. (éd.) (2004), Langages 156 : Effacement énonciatif et discours rapportés.

RASTIER F. (2011), La Mesure et le Grain. Sémantique de corpus, Paris : Honoré Champion.

RINCK F. (2006), L'article de recherche en Sciences du Langage et en Lettres : figures de l'auteur et identité disciplinaire du genre, Thèse de doctorat sous la direction de F.Grossmann et F. Boch, Université Stendhal, Grenoble. [http://w3.u-grenoble3.fr/lidilem/labo/file/theseFannyRinck.pdf]

RINCK F. (2010), "L'analyse linguistique des enjeux de connaissance dans le discours scientifique », in GROSSMANN (2010), 427-450.

ROWLEY-JOLIVET E. (1997), La communication scientifique orale : étude des caractéristiques linguistiques et discursives d'un genre, Thèse de doctorat sous la direction de M. Perrin, Université de Bordeaux.

ROWLEY-JOLIVET E. \& CARTER-THOMAS S. (2005), « The rhetoric of conference presentation introductions : Context, argument and interaction », International Journal of Applied Linguistics 15 (1), 45-70.

Salager-Meyer F., Alcaraz-Ariza M. Ángeles \& PABÓn M. (2007), « The Prosecutor and the Defendant: Contrasting Critical Voices in French and English Written Academic Book Reviews », in FLøTTUM (2007), 109-128.

SAlager-Meyer F., Alcaraz-Ariza M. Á. \& PABÓn M. (2010), «How's who? Protagonists' identification in scholarly book reviews (1890-2008) », in BoCH et RINCK (2010), 59-78.

SAussure F. de (1967), Cours de linguistique générale, publié par C. Bally et A. Séchehaye [1916], édition critique de T. de Mauro, Paris : Payot.

SAVATOVSKY D. (éd.) (1995), Langages 120: Les savoirs de la langue : histoire et disciplinarité.

SERVOS J. W. (1993), « Research Schools and their histories », Osiris 8, 3-15.

SOFIA E. (2011), «Qu'est-ce qu'un brouillon en sciences du langage ? Notes préalables à une édition numérique des manuscrits de F. de Saussure », Cahiers Ferdinand de Saussure $63,11-27$.

SofiA E. (2012), «Comment écrire pour transmettre ? Modalités argumentatives chez Saussure », in FENOGLIO (2012), 147-160.

SOFIA E. (2015), Collation Sechehaye du troisième cours de linguistique générale de Ferdinand de Saussure (1913). Transcription, édition, introduction et notes d'E. Sofía, Leuven : Peeters.

SuOMElA-SALMi E. (2009), «Résumés des articles scientifiques : conventions disciplinaires, langagières ou pragmatiques ? » in J.-M. Defays et A. Englebert (éds.), Principes et typologie du discours universitaire, Paris : L'Harmattan, 297-312.

Swales J. (1990), Genre Analysis: English in Academic and Research Settings, Cambridge : Cambridge University Press. 
Swales J. (2004), Research Genres. Exploration and Applications, Cambridge : Cambridge University Press.

SwALES J. \& VAN BON S. (2007), «Similarities and Differences in French and English EAP Research Article Abstracts: The case of Asp », in FLøTTUM (2007), 260-276.

TESTENOIRE P.-Y. (2010), «Genèse d'un principe saussurien : la linéarité », Recto-Verso 6 : Genèse de la pensée II, cheminements et procédures. [http://www.revuerectoverso.com/spip.php?article179]

TESTENOIRE P.-Y. (2013), Ferdinand de Saussure à la recherche des anagrammes, Limoges : Lambert-Lucas.

TESTENOIRE P.-Y. (2015), «Présentation et inventaire des archives de Joseph Vendryes du Collège de France », Études Celtiques 41, 245-270.

TESTENOIRE P.-Y. (2016), "L'intérêt historiographique des manuscrits de travail de linguistes : l'exemple de la linguistique générale de Joseph Vendryes », $5^{e}$ Congrès Mondial de Linguistique Française - CMLF'16, Institut de Linguistique Française EDP sciences. [http://dx.doi.org/10.1051/shsconf/20162705003]

TORNi D. \& TRABAL P. (2006), «Le résumé de communication comme objet sociologique. Une analyse thématique, ontologique et littéraire à l'aide du logiciel Prospéro », in D. Demazière, C. Brossaud, P. Trabal \& K. Van Meter (éds.), Analyses textuelles en sociologie. Logiciels, méthodes, usages, Rennes : Presses Universitaires de Rennes, 2379.

Tutin A. (éd.) (2007), Revue Française de Linguistique Appliquée 12 (2) : Lexique et écrits scientifiques.

Tutin A. \& Grossmann F. (éds.) (2013), L'écrit scientifique: du lexique au discours. Rennes : Presses Universitaires de Rennes.

VALETTE M. (2006), « La genèse textuelle des concepts scientifiques. Étude sémantique sur l'œuvre du linguiste Gustave Guillaume », Cahiers de Lexicologie 89, 125-142.

VINCENT F. (2013), Le premier cours de linguistique générale professé par Ferdinand de Saussure à Genève. Cours I et sténographie CAILLE - Transcriptions et commentaires, thèse de doctorat sous la direction de D. Ducard, Paris-Est Créteil.

WAQuet F. (2003), Parler comme un livre. L'oralité et le savoir (XVIe-XXe siècle), Paris : Albin Michel.

WAquet F. (2008), Les enfants de Socrate. Filiation intellectuelle et transmission du savoir. XVIIe-XXIe, Paris : Albin Michel.

WAQUET F. (2010), Respublica academica. Rituels universitaires et genres du savoir (XVIIXXIe siècle), Paris : PUPS.

WAQUET Françoise (2015). L'ordre matériel du savoir. Comment les savants travaillent. XVIe-XXe siècle. Paris : CNRS. 